\title{
The Research and Summaries about Some Questions of Learning Style
}

\author{
Ya-dong $\mathrm{LI}^{1, \mathrm{a},{ }^{*}}$ \\ ${ }^{1}$ Linyi University, Feixian, Shandong, China \\ alydxmf|@126.com
}

Keywords: Learning style, Concept, Classification, Determination, Cultural factors.

\begin{abstract}
The article emphasizes and introduces the researches and summaries on several questions of Learning Style more overall, which includes the concept of Learning Style, the constitutional elements and classification of Learning Style, the determination of Learning Style and cultural factors and the Learning Style and so on.
\end{abstract}

\section{Introduction}

At the earliest, the discussion about learning problems of human can be dated back to B.D. more than 400 years' philosophical thinking about the origin of knowledge which led to Plato and Socrates. But the term of Learning Style was the event which appeared at the middle of 20 centuries. In 1954, Herb The lan used officially the word "Learning Style" at first. Later people began to study Learning Style in depth. It is the Rita \& Kenneth Dunn couples coming from the United State of New York's St John's University who study in this area systematically and scientifically at first.

\section{The Concept of Learning Style}

Learning Style also called learning type or learning way. But the researchers have their own different understanding about the definition and meaning of this concept. At present, the most general and recapitulative definition is that Learning Style refers to the consistent ways which the students responding to stimulation in the process of learning and using stimulation. However other scholars also have their own views. Some think Learning Style is a way of learning and the learning trend, for example, Hunt thinks "Learning Style is the most likely learning way which describes the educational situation where students are, it doesn't refer to what students have learned but how to learn". Some think that Learning Style refers to an attitude, for example, Grash \& Riechmann thinks "Learning Style is the attitude which students towards to study, teachers, peers and class". Some think Learning Style is a kind of method or strategy, for example, Schmeck argues that "Learning Style is a kind of method or strategy, although everyone's learning method is from some special strategies, these strategies are the performance of one's learning type". Malcom and others hold the view that "Learning Style is the method used by students when they encounter problems during their educational experience". Some people regard Learning Style as a series of behavior, such as what Keefee thinks. He considers that "Learning Style is the consistent behavior model which individuals express when they attain educational experience, these behavior is used by learners to perceive learning environment, to interact with environment and as a stable pointer reflecting to learning environment". Some regard Learning Style as a processing method, like what Gregory \& Ward said. He believes that "the teachers' Learning Style and the students' Learning Style are 
actually the one's manner." In addition, there are also some people regarding Learning Style and Cognitive Style as synonyms, or example, Kuchinskas often likes to use "Cognitive Style" to explain a person's way of reflecting to environment and behavior or adaptation. Though researchers hold different views of definition to Learning Style, in general, the following points are universally admitted:

(1)There exists unique in Learning Style. "Everyone has a learning style" "it's such characteristic as a signature" [1].

(2)There is no good or bad in Learning Style itself , each learning style can become the most effective with its own way[1].

(3)There are stability and consistency in Learning Style. It is a habituation learning way.

(4)Learning Style can't be equated with Cognitive Style, it is broader than Cognitive Style, or we can say that the learning style includes cognitive style.

(5)Taking teaching problems into consideration, if the students' learning styles match to teachers' teaching styles, they will learn better.

\section{The Constitutional Elements and Classification of Learning Style}

The constitutional elements and classification of Learning Style are the most important and the most key part in the Learning Style research. It is one of the main basis which is used to identify and determine the students' learning style. There are lots of theoretical researches on this question; we are focus on the following five categories:

\section{Kolb's Classification of Learning Style}

Kolb's theory bases on the scheme and operational ideas from Piaget, which divides Learning Style by two dimensions including individuals how to perceive information and how to internalize information. He thinks the perception of information can be divide into concrete experiment style and abstract conception style, likewise, the internalization and processing of information can be divided into behavioral experiment style and thinking observation style. Then we get four types of learning by synthesizing the two dimensions: (1) The Divergers Style: its concrete or thinking. The style of students always like asking "why". (2)The Converges Style: It's abstract or behavioral. The style of students always like asking "how". (3)The Assimilators Style: It's abstract or thinking. The style of students always like asking "what".(4)The Accommodators Style: It's concrete or behavioral. The style of students always like asking "what if".

\section{Dunn \& Dunn's Constitutional Elements and Classification of Learning Style}

In 1974, Dunn\& Dunn couple started trying to construct the learning style models. Initially, they divided learning style into four categories of twenty-four elements in 1978,later they increased the fifth major category of three psychology elements in 1986.At present, this is the most widely used model in the U.S. schools. The main contents include the following five categories: The first category is environmental elements. It includes the preference for learning environment which is quiet or noisy and the preference for light which is intense or faint and the preference for temperature which is high or low and the preference for sitting posture which is normal or casual. The second category is emotional elements. It includes the motivation of self inspiration and the motivation of parents' inspiration and the motivation of teachers' inspiration and lacking of learning motivation and the strength or weak of learning persistence and the strong or weak sense of learning responsibility and the preference for the degree on 
organization of learning content and so on. The third category is social elements. It includes loving to learn independent and loving to learn together and loving to learn with adults and loving to learn with all kinds of different people. The fourth category is physiological elements. It includes the like of acoustic stimulus and the like of visual stimulus and the like of kinesthetic stimulus, in addition, it includes whether love eating snacks or not when studying, and includes in the early morning making the best learning effect or in the morning making the best learning effect or in the afternoon making the best learning effect or in the evening making the best learning effect as well as whether love activating or not when studying. The fifth category is psychological elements. It includes the analysis and synthesis of brain and the preference for the left and right hemispheres of brain as well as meditation and impulsion and other factors.

\section{Gregorc's Classification of Learning Style}

The curriculum of the United States Connecticut States and its teaching professor Gregorc classify Learning Style by two dimensions:(1)How people perceive the world, that is mean, people's perceptual preference.(2)How people understand the world, that is to say, people's organizational preference. Perceptual preference refers to the concrete and abstract aspects whose ways to receive information: the concrete aspect refers to acquire information by the feeling of stimulating physical characteristic; however, the abstract aspect refers to acquire information by the intuition and imagination and so on. Similarly, organizational preference refers to the sequential and random aspects which process information. The learners can be divided into concrete style and abstract style by the way of receiving information (perceptual preference); likewise, by the way of processing information (organizational preference) the learners can be divided into ordered style and out-of-order style, or sequential style and random style. Then according to the learners on the different tendency degree which is concrete and abstract as well as ordered and out-of-order, they can be divided into four different styles which are Concrete Sequential (referred to as CS) and Concrete Random (referred to as RS) and Abstract Sequential (referred to as AS) and Abstract Random (referred to as AR).

\section{Keefee's Elements of Learning Style}

In 1979, Keefee, who was the chairman of National Association of Secondary School Principle (referred to as NASSP), synthesized a variety of learning style model theories, and then extracted the three dimensions of numbers of elements, which based on factors analysis of these theories.

\section{The First Aspect is Cognition Style which includes the Following Elements:}

(1)The accepting style: a)The preference for perceptual channel including visual and hearing and kinesthesis; b)The dependency and the field independence; c) Scanning and focusing; d)Functional fixation and flexibility; e) The tolerance of unreal experience; f) The degree of automation (or the capacity of operating simple and repetitive tasks); g)the perceptual and rational activities.

(2)The conceptual and keeping style: a) The conceptual speed (that is to say the expression of the fast impulsion or slow meditation during the procession of information processed); b) The conceptual style; c) The width or narrowness of classification; d) The complexity and simplicity of cognition; e) The convergence and the divergence. 


\section{The Second Aspect is Emotion Style which Consists of the Following Elements:}

(1)The attention style: a) The rational level; b) Curiosity; c) Perseverance; d) The level of anxiety; e) The endurance of frustration.

(2)The expectation and motivation style: a) The control points; b) The motivation of achievement; c) The motivation of self-realization; d) Imitation; e) The risk and the caution; f) The competition and cooperation; g) The level of aspiration; h)The response for the intensification; i) The motivation of sociality; j) The individual interests.

The third aspect is physiological style which consists of the following elements: (1) Male and female behavior; (2) The health-related behavior; (3) The rhythm of time; (4) Activity behavior; (5) Environmental factors (including the preference for the sound and light and temperature).

\section{Felder-Silverman's Classification of Learning Style Dimensions}

When discussing to the relationship between Learning Style and Teaching Style, Felder\& Silverman points it out that it does not say to teach depending on the performance of students (Learning Style) completely, but to strive for a balance between teaching methods and learning style. The so-called equilibrium state is that teaching in the way of students' preference in some way makes students feel comfortable and willing to learn; But at the same time, teachers teach in the way which some students don't like, which provides the practical opportunities and feedback for students to apply those methods that they are not suitable for at first to think and solve the problems, and which makes them apply these ways and methods efficiently and expertly at last (Felder, 1998) [2].

They classify Learning Style depend on the following five aspects:

(1)Which kind of styles of information students prefer to receive: a) Perceptual style; b) Intuitive style.

(2)Which kind of feeling channels of perceptual information can be received efficiently: a) Visual style; b) Words style.

(3)Which kind of formal information the students are the most suitable for: a) Inductive style; b) Deductive style.

(4)The students how to process information: a) Active style; b) Thinking style.

(5)The students how to understand the procession: a) Sequential style; b) Integrated style.

\section{The Determination of Learning Style}

Hill believes it that "for the students having normal mental capacity, as long as the teaching media and methods match their cognitive styles, $90 \%$ of them can grasp $90 \%$ of teaching contents at the end of teaching". Therefore, there exists the important meaning of teaching on identifying and determining the students' Learning Style.

The determination of Learning Style mainly adopts the method called questionnaire survey. Nowadays there are many questionnaires about Learning Style, for the convenience of understanding, now let's make a brief introduction from two angles.

\section{The Determination of Learning Style Aiming at Learners in Different Age}

At present, it is supposed to have the corresponding Learning Style questionnaires aiming at learners from pupils to adults. Nowadays the most popular survey tool of Learning Style is Dunn \& Dunn couple's "Learning Style Inventory" [3] (referred to as LSI) whose applicable objects are the students of grade three to twelve, at the same time, 
they also have the Learning Style Inventory whose version aims at adults named Productivity Environmental Preference Survey. What's more, Grasha-Riechmann's Learning Style Inventory is applicable to senior high school students and undergraduates.

\section{The Periods of Determination of Different Elements in Learning Style}

In the view of elements in survey, the questionnaires about Learning Style can be divided into two categories: one is determination of comprehensive elements which reflects the comprehensive characteristics of learners' Learning Style, the other is determination of single element which reflects a characteristic of learners' Learning Style. The survey questionnaires about comprehensive elements mainly include Dunn couple's Learning Style Inventory (referred to as LSE) and Keefee's Learning Style Profile (referred to as LSP) and Gregore \& Ward's Transactional Ability Inventory (referred to as TAI,1977) [4].

The survey questionnaires about single element mainly include:(1) Hermen Witkin's The Group Embedded Figures Test (referred to as GEFT,1971),the test is used to test the capacity which learners find out a simple pattern hidden in complex patterns, called the analyticity - comprehensiveness of the information processed or the dependence independence of the field.(2)David Hunt's Paragraph Completion Method (referred to as PCM,1978),it is used to test the conceptual level of learners which is high or low, namely, the degree on organization of learning contents of learners. (3)The corresponding survey questionnaires about testing mental locus of control scale. The locus of control refers to it that the power motivating individuals' behavior derives from the individual internal factors (called internal control) or the individual external factors (called external control). The survey questionnaires about measuring locus of control mainly include Rotter's Internal - External Locus of Control Scale (1996) and Crandall's Intellectual Achievement Responsibility Questionnaire (referred to as IRA,1965).(4)The corresponding survey questionnaires about testing the level of anxiety. The anxious level refers to the level which students feel nervous and anxious when studying. The corresponding survey questionnaires about testing the anxious level mainly include Charles \& Spielberger's State - Trait Anxiety Inventory (referred to as STAI, 1997) and John Gillis's Anxiety Scale for Children (1980).

\section{The Cultural Elements and Learning Style}

Hilliard defined Learning Style as the persistent behavior individual or group tending to habituation - the most obvious peculiarity when people acquire things, which is a characteristic way(1998).Different from discussing individual Learning Style merely by people formerly, Hilliard thinks every group even every culture has its special Learning Style. Jones \& Fennimore argues that" every culture has its thinking habits and learning resource and knowledgeable background, all of these affect our learning and knowledge, accordingly, the curriculum the children under each cultural background learning and the education the children under each cultural background receiving should come from the culture where children are (1990)".Irvine \& York generalizes some cultural factors affecting individual Learning Style as follow:

The degree on self - identify with culture the one belonging to; the condition of social,economy;sex;parentingbehavior;familyenvironment;nationalsubculture;discrimi natedexperience; the resident time in the U.S.; the degree of being assimilated by mainstream culture and so on (1995). 
In addition, the degree which is assimilated by mainstream culture having influence on individual Learning Style, Hilliard explains that the people who spend most of the time in staying in the culture of core which they belong to and those who the whole day get along with people belonging to the same culture with themselves are most likely to show the Learning Style of their culture; And that the people who are in a culture of outside and those who contact with the central culture seldom or even hardly are the least to show the Learning Style of this culture; And that people who are in a culture of margin and those who switch back and forth between the core and the outside have the ability called code switching, this sort of people can not only adapt more than one kind of learning style, but also cope with their core culture and social mainstream culture successfully (1991) [5].

\section{Summary}

In the past two decades, many scholars have carried out many comparative researches on the Learning Style of African American immigrants and Latinos and native Americans. However, some scholars like Dunn and Beaudry \& Klaves put forward objection to mixing cultural elements into Learning Style, they argue that Learning Style is a pure individual phenomenon (1989).Aiming at numerous critical opinions, as Hilliard (1989) and Irvine \& Yord (1995) and other researchers explain it, they warn people against accepting the opinion about cultural Learning Style blindly. Teachers are supposed to have a deep understanding of the culture students belonging to as well as know the condition of each student comprehensively and exactly. The human learning problems are always new in all ages, the research on Learning Style is in-depth and extensive gradually, which makes human know ourselves more and more distinct and comprehensive. It is helpful to enhance human learning ability further as well as to improve human overall quality.

\section{Reference}

[1] Jeannette-vos, Gordon Dryden. The Learning Revolution [M]. Translation by GuRuirong and others. Shanghai: Shanghai San Lian Book Store, 1998.

[2] Gao Xiyuan. The teaching understanding from the perspective of aesthetics [D].Huazhong Normal University, 2012.

[3] Zhang Qiong. Practice ability train-oriented study of the knowledge teaching reform [D]. Huazhong Normal University, 2011.

[4] Chen Na. The conceptual analysis of complete experience and its enlightenment to the teaching of kindergarten [J]. Studies in Preschool Education, 2013(05).

[5] Li Haoquan, LiShanyi. Study on the learning style from the perspective of Chinese traditional thinking [J]. Educational Science Research, 2013(08). 